\title{
Massive stellar models: rotational evolution, metallicity effects
}

\author{
Sylvia Ekström ${ }^{1}$, Cyril Georgy ${ }^{1}$, Georges Meynet ${ }^{1}$, \\ André Maeder ${ }^{1}$, and Anahí Granada ${ }^{1,2}$ \\ ${ }^{1}$ Geneva Observatory, University of Geneva \\ Maillettes 51 - Sauverny, CH-1290 Versoix, Switzerland \\ ${ }^{2}$ Instituto de Astrofísica de La Plata, Universidad Nacional de La Plata, \\ Paseo del Bosque S/N, La Plata, Buenos Aires, Argentina
}

\begin{abstract}
The Be star phenomenon is related to fast rotation, although the cause of this fast rotation is not yet clearly established. The basic effects of fast rotation on the stellar structure are reviewed: oblateness, mixing, anisotropic winds. The processes governing the evolution of the equatorial velocity of a single star (transport mechanisms and mass loss) are presented, as well as their metallicity dependence. The theoretical results are compared to observations of B and Be stars in the Galaxy and the Magellanic Clouds.
\end{abstract}

Keywords. stars: evolution, stars: rotation, stars: winds, outflows, stars: mass loss, stars: emission-line, Be

\section{Introduction}

Why care about rotation? Just because stars do rotate! A look at the velocity distribution established by Huang \& Gies (2006) reveals that the peak in the probability density occurs at $v_{\text {eq }} \simeq 200 \mathrm{~km} / \mathrm{s}$, which represents a ratio $v / v_{\text {crit }} \simeq 0.5-0.6$, i.e. a substantial fraction of the keplerian velocity.

The effects of rotation in stars were studied since the works of von Zeipel and Eddington in the years 1924-1925. In the end of the 60s, they were included in polytropic or simplified stellar models (Roxburgh et al. 1965; Roxburgh \& Strittmatter 1966; Faulkner et al. 1968; Kippenhahn \& Thomas 1970; Endal \& Sofia 1976). About 30 years later, stellar models became more sophisticated and also benefited from the inclusion of rotational effects (Pinsonneault et al. 1989; Deupree 1990; Fliegner \& Langer 1994; Chaboyer et al. 1995; Meynet 1996).

Since the end of the 90s, more or less extended grids of rotating stellar models were computed (Langer et al. 1997; Meynet \& Maeder 1997; Siess \& Livio 1997; Heger et al. 2000). Those grids showed that the inclusion of the effects of rotation improved the adequation between models and massive stars observations in many aspects:

- the surface abundances of light elements (Heger \& Langer 2000; Meynet \& Maeder 2000);

- the predicted surface velocities in clusters (Martayan et al. 2006a,b; Meynet \& Maeder 2000);

- the blue- to red-supergiants number ratio in the SMC (Maeder \& Meynet 2001);

- the WR populations number with metallicity (Meynet \& Maeder 2003, 2005; Vink \& de Koter 2005);

- the rotation rates of pulsars (when strong coupling is assumed, Heger et al. 2005);

- the SN types and GRB progenitors (Meynet \& Maeder 2005; Yoon et al. 2006; Georgy et al. 2009). 
Roughly summarised, rotating stars are expected to present

(a) a modified gravity:

- the surface characteristics become dependent on the colatitude considered;

- there is a mass loss enhancement and anisotropy;

(b) chemical species and angular momentum transport mechanisms:

- the behaviour on the HR diagram is modified;

- the nucleosynthesis is altered;

- there is a surface enrichment;

- the mass loss is modified;

- the rotation profile evolves during the star life, becoming steeper (when no magnetic fields are considered).

We will go through the different points in this review.

\section{Surface characteristics}

\subsection{Gravity and shape}

Because of rotation, the effective gravity is modified, becoming a function of the rotation velocity $\Omega$ and of the colatitude $\theta$ of the star:

$$
\vec{g}_{\mathrm{eff}}=\vec{g}_{\mathrm{eff}}(\Omega, \theta)=\left(-\frac{G M}{r^{2}}+\Omega^{2} r \sin ^{2} \theta\right) \vec{e}_{r}+\Omega^{2} r \sin \theta \cos \theta \vec{e}_{\theta} .
$$

We immediately see that at the pole $\left(\theta=0^{\circ}\right)$ the effective gravity is just the gravitation acceleration $-G M / r^{2}$. At the equator $\left(\theta=90^{\circ}\right)$ the centrifugal force adds a sustaining term $\Omega^{2} r \sin ^{2} \theta$. In these two cases, the effective gravity is still radial, while at intermediate $\theta$, the term $\Omega^{2} r \sin \theta \cos \theta$ does not vanish and implies that the effective gravity is no more radial.

In the frame of the Roche model, the maximal oblateness allowed when the star rotates at the critical velocity $\dagger$ is $R_{\text {eq,crit }}=1.5 R_{\text {pol,crit }}$.

Recently, interferometry has allowed to determine the deformation of fast rotating stars. A first evaluation of the oblateness of Achernar by Domiciano de Souza et al. (2003) showed a larger ratio $R_{\text {eq }} / R_{\text {pol }}$ than the one allowed in the Roche model. However, more recent observations of the same star (Vinicius et al. 2006; Carciofi et al. 2008) have revised this ratio and found a lower value, more compatible with these theoretical expectations.

\subsection{Flux and effective temperature}

Since the flux is related to the effective gravity (von Zeipel 1924; Owocki et al. 1996, 1998; Maeder 1999), it becomes also dependent on colatitude:

$$
\vec{F}=\vec{F}(\Omega, \theta) \simeq-\frac{L}{4 \pi G M^{\star}} \vec{g}_{\mathrm{eff}}(\Omega, \theta)
$$

with $M^{\star}=M\left(1-\frac{\Omega^{2}}{2 \pi G \rho_{m}}\right)$ the so-called reduced mass which takes into account the reduction of the gravitational potential by rotation $\left(\rho_{\mathrm{m}}\right.$ is the mean density inside the considered isobar).

$\dagger$ The critical velocity is reached when $\vec{g}_{\text {eff }}$ vanishes because the centrifugal force at the equator counterbalances the gravity exactly (see Section 3.2). 


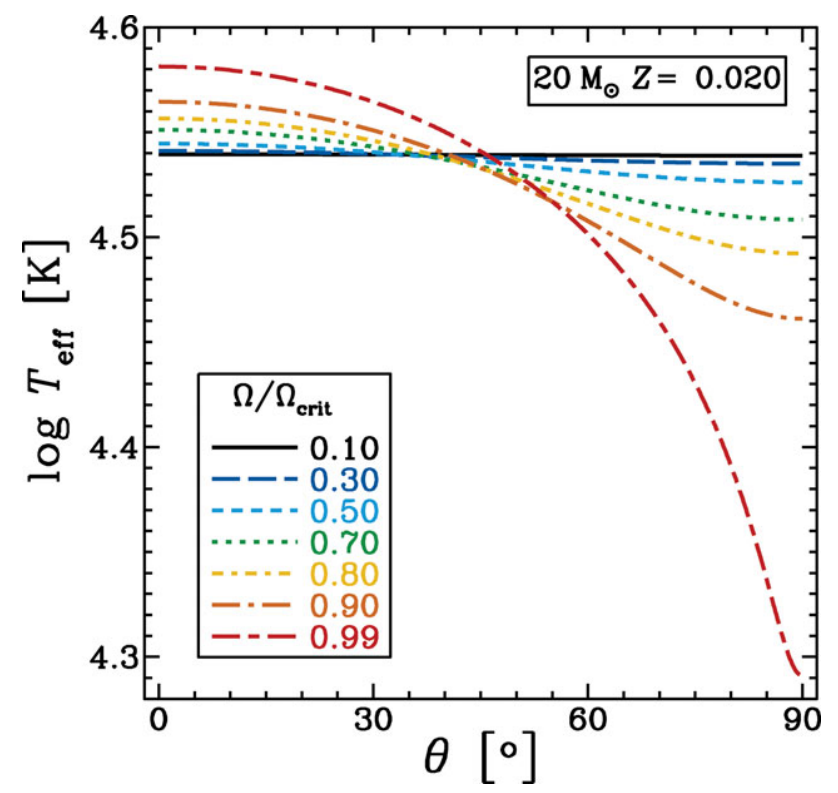

Figure 1. Variation of the effective temperature with the colatitude for various initial rotation rates (figure from Ekström et al. 2008).

The Stefan-Boltzmann law $F=\sigma T^{4}$ implies a dependence on the colatitude for the effective temperature as well:

$$
T_{\mathrm{eff}}=T_{\mathrm{eff}}(\Omega, \theta)=\left[\frac{L}{4 \pi \sigma G M^{\star}} \vec{g}_{\mathrm{eff}}(\Omega, \theta)\right]^{1 / 4} .
$$

Recent interferometric observations, like for example Monnier et al. (2007) on Altair or Zhao et al. (2009) on $\alpha$ Cephei and $\alpha$ Ophiuchi, have provided a possibility to test this relation. Let's have a close look at Altair: the rotation rate $\Omega / \Omega_{\text {crit }}$ of this star is evaluated between 0.90 and 0.92 , and the temperature difference between the pole and the equator is between 1.19 and 1.32. For such a rotation rate, the theoretical models (cf. Fig. 1) predict a difference of 1.26-1.33, in good agreement with the observational value.

\subsection{Mass loss}

According to the works of Owocki \& Gayley (1997), Maeder \& Meynet (2000) and Petrenz $\&$ Puls (2000), rotation enhances the mass loss by a factor:

$$
\frac{\dot{M}(\Omega)}{\dot{M}(0)}=\left[\frac{\left(1-\Gamma_{\mathrm{Edd}}\right)}{\left(1-\frac{\Omega^{2}}{2 \pi G \rho_{m}}-\Gamma_{\mathrm{Edd}}\right)}\right]^{\frac{1}{\alpha}-1}
$$

where $\Gamma_{\mathrm{Edd}}$ is the Eddington factor, i.e. the ratio of the luminosity of the star to the Eddington luminosity $L_{\mathrm{Edd}}=\frac{\kappa_{\mathrm{s}}}{4 \pi c G M}$, with $\kappa_{\mathrm{s}}$ the electron-scattering opacity.

It also changes the geometry of the mass flux (Maeder 2002, 2009), which is no longer constant on the whole surface of the star. The mass loss by surface unit at a given latitude $\theta$ follows the relation:

$$
\frac{\mathrm{d} \dot{M}(\theta)}{\mathrm{d} \sigma} \sim A(\alpha, k)\left(\frac{L}{4 \pi G M^{\star}}\right)^{\frac{1}{\alpha}-\frac{1}{8}} \frac{g_{\mathrm{eff}}(\theta)^{1-\frac{1}{8}}}{\left(1-\Gamma_{\Omega}(\theta)\right)^{\frac{1}{\alpha}-1}}
$$



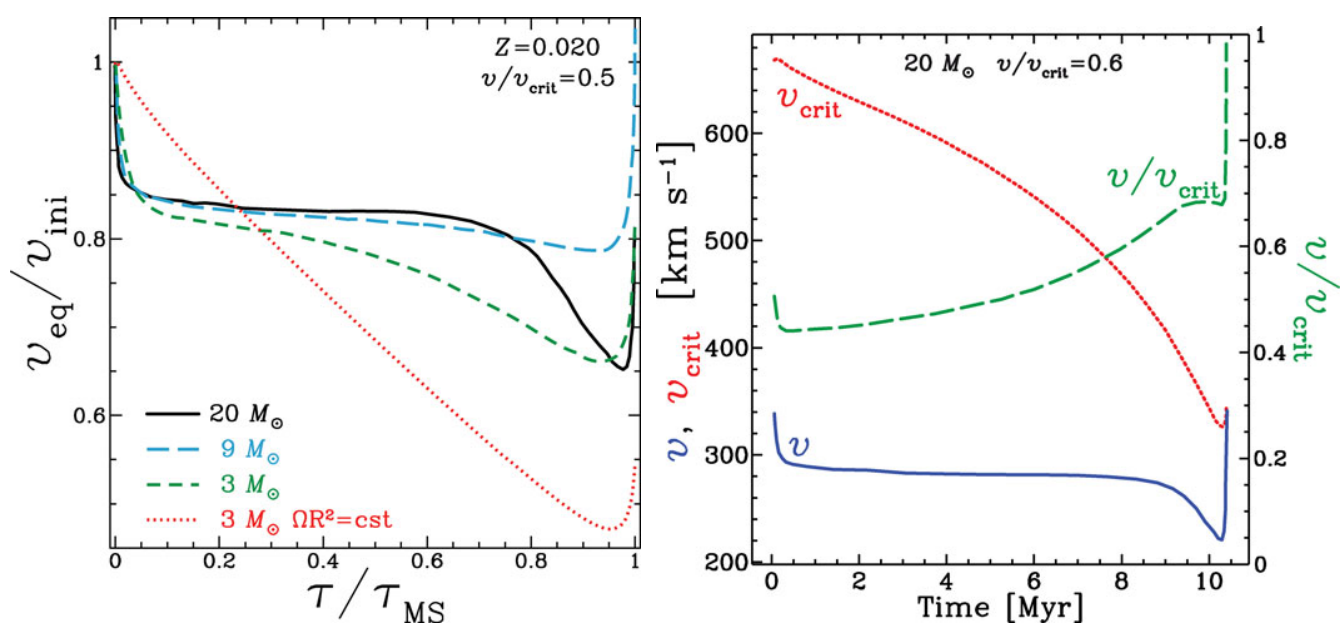

Figure 2. Left: evolution of the equatorial velocity (normalised to the initial velocity) during the main sequence for different mass domains. Right: evolution of the equatorial velocity, the critical velocity and the ratio $v / v_{\text {crit }}$ during the main sequence.

where $A(\alpha, k)=(k \alpha)^{1 / \alpha}\left(\frac{1-\alpha}{\alpha}\right)^{(1-\alpha) / \alpha}$ is a function of the force multiplier parameters which characterise the stellar opacity.

If we consider that $A=\mathrm{cst}$, a star rotating for example at $\Omega / \Omega_{\text {crit }}=0.95$ will have $\dot{M}(\mathrm{pol})=3.2 \dot{M}(\mathrm{eq})$. However, if one accounts for the change of the force multiplier parameters, i.e. a variation in the opacity regime caused by the drop of $T_{\text {eff }}$ at the equator, the equatorial mass loss can be enhanced at a given point, driving the formation of a decretion disc (Owocki 2004).

On the observations side, interferometry again sheds a new light in this topic, showing features that can be interpreted as polar enhanced winds (Kervella \& Domiciano de Souza 2006; Meilland et al. 2007), as well as discs around active stars (Meilland et al. 2007; Schaefer et al. 2010).

The geometry of the wind may leave an imprint on the circumstellar medium. There are some indications of asymmetry detected in spectropolarimetry observations of some supernovae (see for example SN 2007rt by Trundle et al. 2009). This aspect is actually under study with 2- and 3D simulations and will be the subject of a future paper (Walder et al. in prep).

\section{Rotational evolution}

\subsection{Two competing processes}

The evolution of the surface velocity of a star is the result of the competition between two processes:

(a) the mass loss, which removes angular momentum at the surface and thus decelerates the rotational velocity;

(b) the transport of angular momentum inside the star, which brings some internal angular momentum to the surface (mainly through the meridional circulation) and may counterbalance the loss by the winds.

Both processes are dependent on the mass of the star: the more massive the star, the stronger winds and larger meridional currents it experiences (see Fig. 2, left). At solar metallicity typically, around $20 M_{\odot}$ and above, the winds contribution wins so the star 


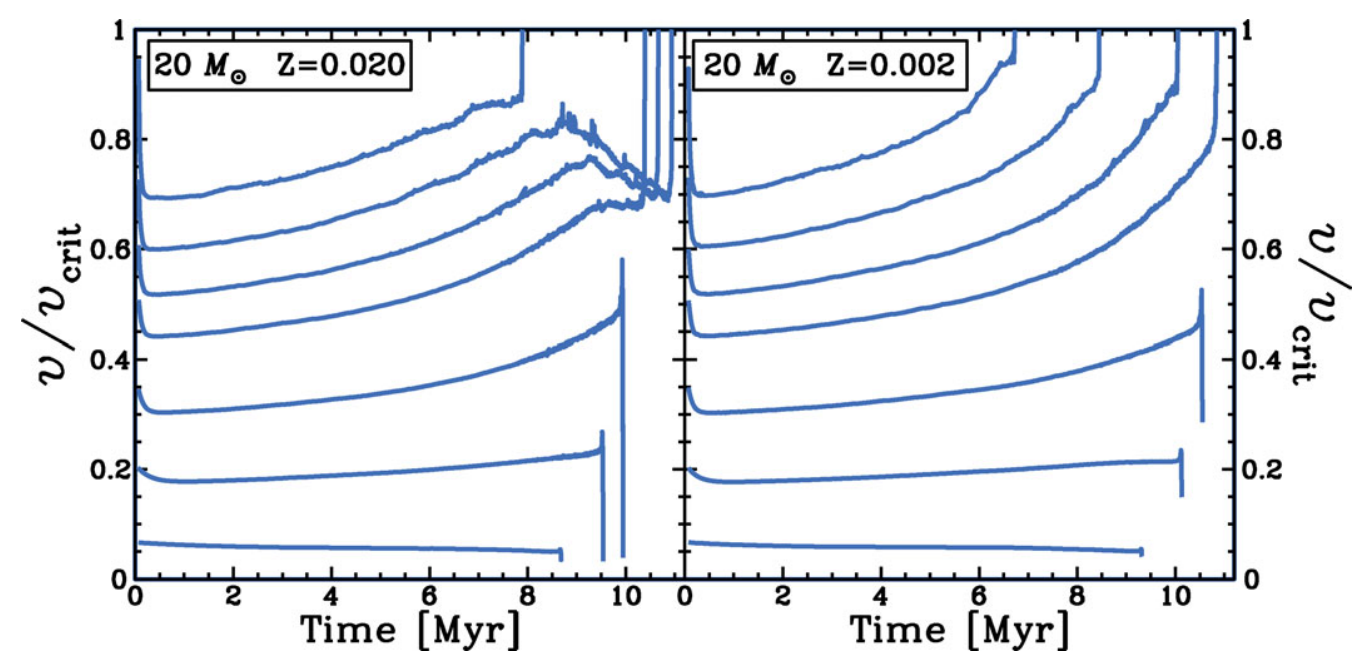

Figure 3. Evolution of the $v / v_{\text {crit }}$ ratio during the main sequence for $20 M_{\odot}$ models at $Z=0.020$ and $Z=0.002$.

decelerates during the main sequence (MS). Around $9 M_{\odot}$, both processes are in equilibrium so the surface velocity remains almost constant during the largest part of the MS. Around $3 M_{\odot}$, there are almost no mass loss through winds, but the core-envelope coupling through the meridional circulation is so weak that the surface velocity decreases during the MS. Note that recent observations by Huang et al. (2010, see also Poster S1-05 in these proceedings) show a quicker deceleration of the less massive of the B stars than predicted by current theoretical models, more compatible with a regime of local angular momentum conservation $\Omega R^{2}=$ cst.

\subsection{Critical limit}

One thing is the evolution of the surface velocity, another thing is the evolution of the ratio to the critical limit $v_{\text {crit }}=\sqrt{\frac{2}{3} \frac{G M}{R_{\mathrm{pol}}}}$. During the MS, the mass may be reduced by mass loss mechanisms, and the radius steadily inflates, so the critical limit drops, as shown in Fig. 2, right. The result is that although the surface may decelerates, the star may encounter the critical limit at a moment in its main sequence lifetime. Comparing the left panels of Fig. 3 and 4, we see that the conditions to reach the critical limit during the MS are met if the star has not a too high mass, and also if it is not a too slow rotator at birth. For each mass domain, there is a minimal initial $v / v_{\text {crit }}$ ratio allowing for the reaching of the critical limit.

Once at the critical limit, the star remains close to it. It experiences phases of mass ejection (slowing the surface below the critical value) followed by quiescent phases, during which it slowly re-accelerates toward the critical limit.

It is possible now for theoretical models to evaluate the mass ejected in the form of a disc (see Poster S1-03 by Georgy et al. and S1-06 by Krtička et al. in these proceedings). This 'mechanical' mass loss seems to occur at a lower rate than the one that can be measured around Be stars (Rinehart et al. 1999; Stee 2003). However, in the models, the mechanical mass loss is averaged on a much longer timestep than the period of mass ejection observed in Be stars. The instantaneous mass-loss rate is expected to be higher than the average one given by the models. 


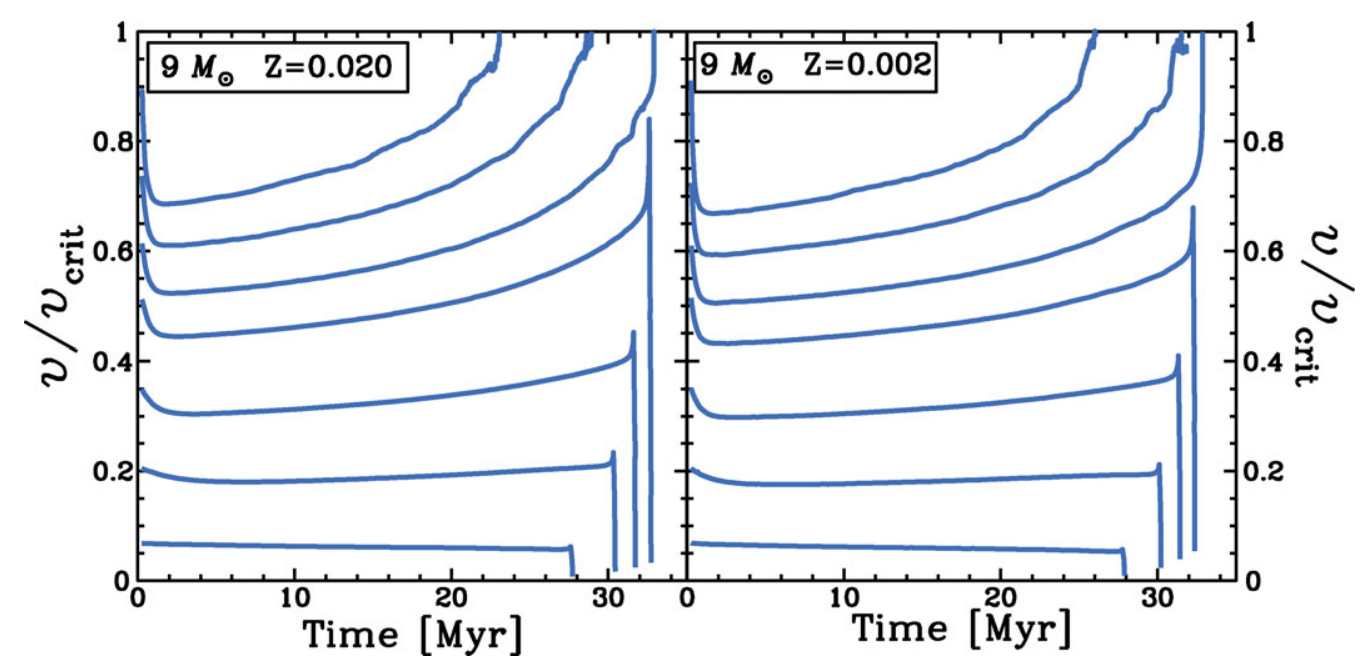

Figure 4. Evolution of the $v / v_{\text {crit }}$ ratio during the main sequence for $9 M_{\odot}$ models at $Z=0.020$ and $Z=0.002$.

\subsection{Metallicity effects}

Both the radiative mass loss rate and the strength of the meridional circulation depend on the metallicity. A low metallicity reduces both mechanisms: there are less metal lines to interact with the photons and form a radiatively driven wind and the greater compactness of the star reduces the amplitude of the meridional circulation.

The net effect on the evolution of the equatorial velocity depends strongly on the mass domain considered: for the most massive stars, the reduction of the winds is the dominant effect, so the stars reach more easily the critical limit (see Fig. 3). On the contrary, for less massive stars for which the winds are not too strong anyway, the reduction of the meridional circulation is dominant, so the stars have more difficulties to reach the critical limit (see Fig. 4).

\subsection{Be phenomenon}

Fast rotation is supposed to be linked with the Be phenomenon. It is however not yet clear whether it may explain by itself the origin of the equatorial disc observed around Be stars. Observations show a ratio $v / v_{\text {crit }} \approx 0.70-0.80$ (Porter 1996; Chauville et al. 2001; Tycner et al. 2005). Maybe this ratio is true, but there may also be alternative explanations: first, as shown by Townsend et al. (2004), there is a saturation effect in the widening of the lines by rotation, so the true velocity may be underestimated. Second, the Be phenomenon seems to consist of ejection phases followed by quiescent phases during which the stars may rotate with a lower rate.

Observations show a metallicity trend in the appearance of the Be phenomenon (see for example Maeder et al. 1999 or Wisniewski \& Bjorkman 2006). Two scenarios are evoked to explain the fast rotation of the Be stars: the binary channel (see for example McSwain \& Gies 2005) (where the fast rotation arises from the accretion of angular momentum from the mass-donor companion), or the single star channel (Ekström et al. 2008) (where the surface acceleration is a natural evolution due to the core-envelope coupling). Any such scenario should present this metallicity trend to be valid. To study the single star evolution scenario, Ekström et al. (2008) computed 112 stellar models (4 masses, 4 metallicities and 7 rotation rates) with the Geneva code. To get populations 
numbers, we convolved the models with a Salpeter IMF, using the velocity distribution of Huang \& Gies (2006).

A good agreement between the theoretical population ratio $\mathrm{Be} /(\mathrm{B}+\mathrm{Be})$ and the observed one is obtained modulo two adjustments:

(a) the Be phenomenon should appear already at $70 \%$ of the critical velocity;

(b) the velocity distribution should count more fast rotators at birth in low-metallicity environments.

Point $(a)$ can be sustained by several mechanisms. For example, sub-surface convective motions have been shown to be able to give the needed impulse to eject matter from the surface (Maeder et al. 2008; Cantiello et al. 2009), as also would non-radial pulsations do (McSwain et al. 2008; Owocki 2004). The binarity of the star could play a role, here not by spinning-up the star but by adding a gravitational pull allowing to launch the matter in the disc (Kervella et al. 2008). Point (b) presents contradictory observational supports. While Martayan et al. (2007) do find a higher mean $\Omega / \Omega_{\text {crit }}$ ratio in the SMC compared to the LMC and the Galaxy, Penny \& Gies (2009) draw opposite conclusions, finding no clear evidence for any difference in the velocity distribution at different metallicities.

Note that the stellar models have been computed without the effects of magnetic fields, the inclusion of which could change the picture. It may be that taking into account the strong core-envelope coupling brought by the magnetic fields relieves the theoretical prediction from the two aforementioned adjustments.

\section{5. $L B V s$}

Until now, the critical velocity we have considered was determined only by the centrifugal contribution $\vec{g}_{\text {rot }}$ against $\vec{g}_{\text {grav }}(\Omega$-limit). However, a critical point appears whenever $\vec{g}_{\text {tot }}=\vec{g}_{\text {grav }}+\vec{g}_{\text {rot }}+\vec{g}_{\text {rad }}=0$. If $\vec{g}_{\text {rot }}=0$ or is negligible, the star may meet the classical Eddington-limit when $\vec{g}_{\text {rad }}=\vec{g}_{\text {grav }}$. If $\vec{g}_{\text {rad }}$ is negligible, the star may encounter the $\Omega$-limit when $\vec{g}_{\text {rot }}=\vec{g}_{\text {grav }}$ : this describes the first critical velocity considered until now:

$$
v_{\text {crit }, 1}=\sqrt{\frac{2}{3} \frac{G M}{R_{\text {pol,crit }}}}
$$

with $R_{\text {pol,crit }}$ the polar radius when the star is at the critical limit.

If all three terms are significant, the star may meet the $\Omega \Gamma$-limit when $\vec{g}_{\mathrm{tot}}=0$. It means that for a given $\Omega$, there is a maximum luminosity given by:

$$
L_{\Gamma \Omega}=\frac{4 \pi c G M}{\kappa}\left(1-\frac{\Omega^{2}}{2 \pi G \rho_{\mathrm{m}}}\right)
$$

with $\kappa$ the total opacity. Inversely, depending on the Eddington factor of the star, a second critical velocity can be defined:

$$
v_{\mathrm{crit}, 2}=\sqrt{\frac{9}{4} v_{\mathrm{crit}, 1}^{2} \frac{1-\Gamma_{\max }}{V^{\prime}(\omega)} \frac{R_{\mathrm{e}}^{2}(\omega)}{R_{\mathrm{pol}, \mathrm{crit}}^{2}}}
$$

where the quantity $V^{\prime}(\omega)=\frac{V(\omega)}{\frac{4 \pi}{3} R_{\mathrm{pol}, \mathrm{crit}}^{3}}$ is the ratio of the actual volume of a star with rotation $\omega=\Omega / \Omega_{\text {crit }}$ to the volume of a sphere of radius $R_{\text {pol,crit }}$.

Note that for $\Gamma_{\mathrm{Edd}}<0.639, v_{\text {crit }, 2}$ is not defined. Above this value, $v_{\text {crit }, 2}$ becomes smaller than $v_{\text {crit, } 1}$, so the star encounters the $\Omega \Gamma$-limit before the $\Omega$-limit. This could be the case of some known LBVs (Groh et al. 2006, 2009), that present both a high Eddington factor and a high rotation rate. With a $\Gamma_{\text {Edd }} \simeq 0.8$, they are probably meeting their second critical velocity already with $\Omega / \Omega_{\text {crit }} \simeq 0.85-0.9$. 

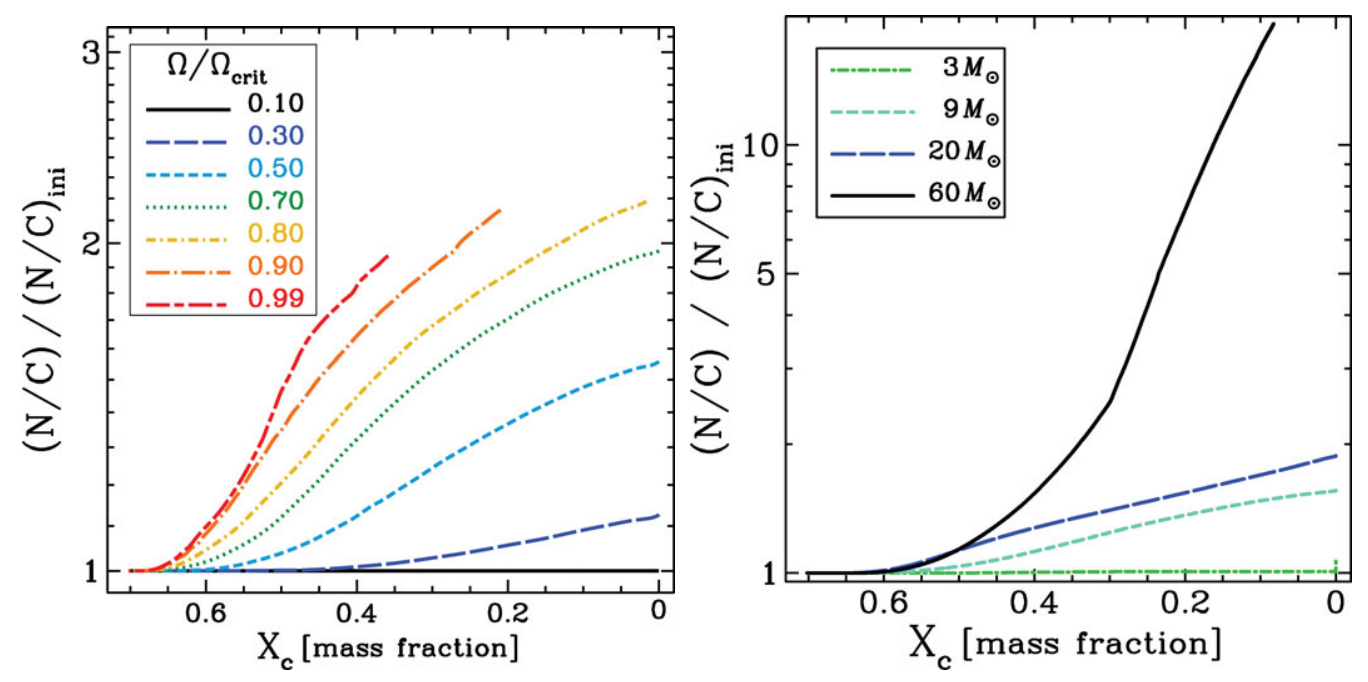

Figure 5. Left: evolution of the surface N/C ratio (normalised to the initial one) during the main sequence (expressed as the central mass fraction of hydrogen $X_{\mathrm{c}}$ ) for $9 M_{\odot}$ models at $Z=0.020$ with varying the initial ratio $\Omega / \Omega_{\text {crit }}$. Right: same as left, but for different mass domains.

\section{Internal mixing}

\subsection{Diffusion of chemical species}

The radiative zones inside a star are supposed to rotate differentially. This causes shear turbulence which in turn drives some turbulent mixing of chemical species. The higher the rotation rate, the stronger is the mixing (see Fig. 5, left).

We can evaluate the diffusion time: $\tau_{\text {mix }} \simeq \frac{R^{2}}{D}$ with $D$ the diffusion coefficient. With a relation between the radius and the mass, we get $\tau_{\text {mix }} \propto M^{-1.8}$ while the main sequence lifetime is $\tau_{\mathrm{MS}} \propto M^{-0.7}$. We see that the more massive the star, the stronger is the mixing, leading to a surface enrichment already on the main sequence (see Fig. 5, right).

\subsection{Metallicity effects}

Low-metallicity stars are more compact than higher-metallicity ones. The meridional currents are less efficient, so the $\Omega$-profile inside the star is steeper. The mixing is thus stronger, while the mixing time is shorter, so we expect a strong surface enrichment on the main sequence.

\subsection{Surface abundances}

The surface abundances are expected to be modified by mixing. A good signature of mixing is the $\mathrm{C}, \mathrm{N}$, and $\mathrm{O}$ abundances: their respective ratios are expected to change, but their sum $\mathrm{C}+\mathrm{N}+\mathrm{O}$ is supposed to remain constant. Only at very low $Z$, primary nitrogen production may occur, leading to a net metallicity increase. A careful abundance analysis made by Przybilla et al. (2010) in the solar neighbourhood shows that the observed mixing follows very well the trend expected from CNO nuclear reactions (see their contribution in the same proceedings). The adequation with stellar models is also good, but the observations are not very constraining yet.

It is very important to keep in mind that the mixing is a function of the rotation rate, of course, but also (as shown above) of the mass, the metallicity and the age, as well as other characteristics as the binarity for example. The study of the rotational mixing in stars should imply a separate analysis, in order to discriminate between several 
parameters. For example, while Hunter et al. (2008), studying field and clusters stars in the SMC, find many outliers from the expected path in the $\mathrm{N} / \mathrm{C}$ vs $v_{\text {rot }}$, a re-analysis by Maeder et al. (2009) of a sub-group of the same stars, restrained to a narrow mass range and belonging to the same cluster (i.e. having the same age), shows that most of the outliers shifted back to the expected path. Most of the outliers left cannot be used to test the rotation-induced mixing, because they are evolved stars or known binaries. For the very few remaining, we indeed need another explanation than pure rotational mixing. There are several possibilities that shall be the subject of further studies, as the influence of magnetic fields inside the star or magnetic braking at the surface. However, the need for a yet unknown process is evoked (see the contribution of Ines Brott in the same proceedings).

\section{Conclusion}

Rotation is linked to many very interesting types of objects as Be stars or LBVs. The inclusion of its effects in theoretical stellar models is essential to understand those objects and their direct environment. While rotating models are in a better adequation with observations, there is still a lot of work to do in order to improve our understanding of the physical processes at work.

Theoretical and numerical developments are continuously on-going, but theory alone is like a car without a driver. Observations are highly needed:

- larger surveys of well identified objects would allow to make a separate analysis of sub-groups in masses, ages, ..., with a significant statistics;

- a larger number of objects studied by interferometric measurements would allow to put constraints on the surface characteristics of the models;

- the progress in asteroseismology should provide constraints on the internal structure of the stars;

- precise observations of the circumstellar environment could help to constrain the mass loss mechanisms, which are a key ingredient in massive stars.

\section{References}

Cantiello, M., Langer, N., Brott, I., de Koter, A. et al. 2009, A $\& A$ 499, 279

Carciofi, A. C., Domiciano de Souza, A., Magalhães, A. M., Bjorkman, J. E. et al. 2008, ApJ (Letters) 676, L41

Chaboyer, B., Demarque, P., \& Pinsonneault, M. H. 1995, ApJ 441, 865

Chauville, J., Zorec, J., Ballereau, D., Morrell, N. et al. 2001, A\&SA 378, 861

Deupree, R. G. 1990, ApJ 357, 175

Domiciano de Souza, A., Kervella, P., Jankov, S., Abe, L. et al. 2003, A\&\&A 407, L47

Ekström, S., Meynet, G., Maeder, A., \& Barblan, F. 2008, A $\& A$ 478, 467

Endal, A. S. \& Sofia, S. 1976, ApJ 210, 184

Faulkner, J., Roxburgh, I. W., \& Strittmatter, P. A. 1968, ApJ 151, 203

Fliegner, J. \& Langer, N. 1994, in: L. A. Balona, H. F. Henrichs, \& J. M. Le Contel (eds.), Pulsation; Rotation; and Mass Loss in Early-Type Stars, IAU Symposium 162, p. 147

Georgy, C., Meynet, G., Walder, R., Folini, D. et al. 2009, A\& A 502, 611

Groh, J. H., Damineli, A., Hillier, D. J., Barbá, R. et al. 2009, ApJ (Letters) 705, L25

Groh, J. H., Hillier, D. J., \& Damineli, A. 2006, ApJ (Letters) 638, L33

Heger, A. \& Langer, N. 2000, ApJ 544, 1016

Heger, A., Langer, N., \& Woosley, S. E. 2000, ApJ 528, 368

Heger, A., Woosley, S. E., \& Spruit, H. C. 2005, ApJ 626, 350

Huang, W. \& Gies, D. R. 2006, ApJ 648, 580 
Huang, W., Gies, D. R., \& McSwain, M. V. 2010, ApJ 722, 605

Hunter, I., Brott, I., Lennon, D. J., Langer, N. et al. 2008, ApJ (Letters) 676, L29

Kervella, P. \& Domiciano de Souza, A. 2006, A\& A 453, 1059

Kervella, P., Domiciano de Souza, A., \& Bendjoya, P. 2008, A\& A 484, L13

Kippenhahn, R. \& Thomas, H.-C. 1970, in: A. Slettebak (eds.), Stellar Rotation, IAU Colloquium 4 (Gordon and Breach Science Publishers), p. 20

Langer, N., Fliegner, J., Heger, A., \& Woosley, S. E. 1997, Nuclear Physics A 621, 457

Maeder, A. 1999, A\&\&A 347, 185

Maeder, A. 2002, A\& $\&$ 392, 575

Maeder, A. 2009, Physics, Formation and Evolution of Rotating Stars, Astronomy and Astrophysics Library (Springer Berlin Heidelberg)

Maeder, A., Georgy, C., \& Meynet, G. 2008, A\& $A$ 479, L37

Maeder, A., Grebel, E. K., \& Mermilliod, J.-C. 1999, A\&A 346, 459

Maeder, A. \& Meynet, G. 2000, A\&A 361, 159

Maeder, A. \& Meynet, G. 2001, A\&A 373, 555

Maeder, A., Meynet, G., Ekström, S., \& Georgy, C. 2009, Communications in Asteroseismology 158,72

Martayan, C., Frémat, Y., Hubert, A.-M., Floquet, M. et al. 2006a, A $\& A$ 452, 273

Martayan, C., Frémat, Y., Hubert, A.-M., Floquet, M. et al. 2007, A\&A 462, 683

Martayan, C., Hubert, A. M., Floquet, M., Fabregat, J. et al. 2006b, A\&SA 445, 931

McSwain, M. V. \& Gies, D. R. 2005, ApJS 161, 118

McSwain, M. V., Huang, W., Gies, D. R., Grundstrom, E. D. et al. 2008, ApJ 672, 590

Meilland, A., Stee, P., Vannier, M., Millour, F. et al. 2007, A\&A 464, 59

Meynet, G. 1996, in: C. Leitherer, U. Fritze-von-Alvensleben, \& J. Huchra (eds.), From Stars to Galaxies: the Impact of Stellar Physics on Galaxy Evolution, ASP-CS 98, p. 160

Meynet, G. \& Maeder, A. 1997, A\&A 321, 465

Meynet, G. \& Maeder, A. 2000, A\&A 361, 101

Meynet, G. \& Maeder, A. 2003, $A \& A 404,975$

Meynet, G. \& Maeder, A. 2005, A\&A 429, 581

Monnier, J. D., Zhao, M., Pedretti, E., Thureau, N. et al. 2007, Science 317, 342

Owocki, S. P. 2004, in: A. Maeder \& P. Eenens (eds.), Stellar Rotation, IAU Symposium 215, p. 515

Owocki, S. P., Cranmer, S. R., \& Gayley, K. G. 1996, ApJ (Letters) 472, L115

Owocki, S. P., Cranmer, S. R., \& Gayley, K. G. 1998, in: A. M. Hubert \& C. Jaschek (eds.), $B[e]$ stars, Astrophysics and Space Science Library 233, p. 205

Owocki, S. P. \& Gayley, K. G. 1997, in: A. Nota \& H. Lamers (eds.), Luminous Blue Variables: Massive Stars in Transition, ASP-CS 120, p. 121

Penny, L. R. \& Gies, D. R. 2009, ApJ 700, 844

Petrenz, P. \& Puls, J. 2000, A\&A A 358, 956

Pinsonneault, M. H., Kawaler, S. D., Sofia, S., \& Demarque, P. 1989, ApJ 338, 424

Porter, J. M. 1996, MNRAS 280, L31

Przybilla, N., Firnstein, M., Nieva, M. F., Meynet, G. et al. 2010, A $\mathscr{E} A$ 517A, 38

Rinehart, S. A., Houck, J. R., \& Smith, J. D. 1999, AJ 118, 2974

Roxburgh, I. W., Griffith, J. S., \& Sweet, P. A. 1965, ZfA 61, 203

Roxburgh, I. W. \& Strittmatter, P. A. 1966, MNRAS 133, 345

Schaefer, G. H., Gies, D. R., Monnier, J. D., Richardson, N. et al. 2010, in: T. Rivinius \& M. Curé (eds.), The Interferometric View on Hot Stars, Rev. Mexicana AyA Conference Series 38, p. 107

Siess, L. \& Livio, M. 1997, ApJ 490, 785

Stee, P. 2003, A\& A 403, 1023

Townsend, R. H. D., Owocki, S. P., \& Howarth, I. D. 2004, MNRAS 350, 189

Trundle, C., Pastorello, A., Benetti, S., Kotak, R. et al. 2009, A\&A 504, 945

Tycner, C., Lester, J. B., Hajian, A. R., Armstrong, J. T. et al. 2005, ApJ 624, 359

Vinicius, M. M. F., Zorec, J., Leister, N. V., \& Levenhagen, R. S. 2006, A\&\&A 446, 643

Vink, J. S. \& de Koter, A. 2005, A\& A 442, 587 
von Zeipel, H. 1924, MNRAS 84, 665

Wisniewski, J. P. \& Bjorkman, K. S. 2006, ApJ 652, 458

Yoon, S.-C., Langer, N., \& Norman, C. 2006, A\& $A$ 460, 199

Zhao, M., Monnier, J. D., Pedretti, E., Thureau, N. et al. 2009, ApJ 701, 209

\section{Discussion}

S. OwockI: I would like to emphasize an important difference between reaching the two critical rotation speeds identified in the Maeder \& Meynet analysis. The first critical speed applies to low luminosity stars like Be stars, and should lead to a circumstellar decretion disk that is ejected mechanically at the equator. But for the second critical speed, which is modified by the radiative forces associated with $\Gamma>0.65$, the mass loss should be mainly over the poles, not equator. In effect, the rapid rotation and associated equatorial gravity darkening forces the stellar luminosity to emerge over a smaller surface area over the poles, so that even if $\Gamma \lesssim 1$ the local flux over the poles can exceed the Eddington value, leading to a radiatively driven, bipolar, prolate mass loss. In short, in low luminosity, $\Gamma<<1$ Be stars, critical rotation leads to the observed equatorial disk, while in $\Gamma \lesssim 1$ LBVs it leads to bipolar nebulae.

O. Chesneau: A comment to Stan: If the mass loss of LBVs is essentially prolate, and directed toward the pole, how can you explain why the environment of LBVs is dominated by ring-like structures (see comment of K. Weis)?

S. OwockI: Well, in slowly rotating LBVs you should get spherical mass loss that will likely appear ring-like. Perhaps Kerstin can comment on how relatively common these rings are vs. bipolar LBVs.

K. WeIs: As Stan already mentioned, LBVs create polar winds which lead to larger number of bipolar nebulae and not only spherical ring nebulae. See my talk on Thursday. 